\title{
A novel stepwise micro-TESE approach in non obstructive azoospermia
}

\author{
Giorgio Franco ${ }^{1}$, Filomena Scarselli ${ }^{2}$, Valentina Casciani ${ }^{2}$, Cosimo De Nunzio ${ }^{3}$, Donato Dente ${ }^{4}$, \\ Costantino Leonardo ${ }^{1 *}$, Pier Francesco Greco ${ }^{2}$, Alessia Greco ${ }^{2}$, Maria Giulia Minasi ${ }^{2}$ and Ermanno Greco ${ }^{2}$
}

\begin{abstract}
Background: The purpose of the study was to investigate whether micro-TESE can improve sperm retrieval rate (SRR) compared to conventional single TESE biopsy on the same testicle or to contralateral multiple TESE, by employing a novel stepwise micro-TESE approach in a population of poor prognosis patients with non-obstructive azoospermia (NOA).

Methods: Sixty-four poor prognosis NOA men undergoing surgical testicular sperm retrieval for ICSI, from March 2007 to April 2013, were included in this study. Patients inclusion criteria were a) previous unsuccessful TESE, b) unfavorable histology (SCOS, MA, sclerahyalinosis), c) Klinefelter syndrome. We employed a stepwise micro-TESE consisting three-steps: 1) single conventional TESE biopsy; 2) micro-TESE on the same testis; 3) contralateral multiple TESE.

Results: SRR was $28.1 \%$ (18/64). Sperm was obtained in both the initial single conventional TESE and in the following micro-TESE. The positive or negative sperm retrieval was further confirmed by a contralateral multiple TESE, when performed. No significant pre-operative predictors of sperm retrieval, including patients' age, previous negative TESE or serological markers (LH, FSH, inhibin B), were observed at univariate or multivariate analysis. Micro-TESE (step 2) did not improve sperm retrieval as compared to single TESE biopsy on the same testicle (step 1) or multiple contralateral TESE (step 3).

Conclusions: Stepwise micro-TESE could represent an optimal approach for sperm retrieval in NOA men. In our view, it should be offered to NOA patients in order to gradually increase surgical invasiveness, when necessary. Stepwise micro-TESE might also reduce the costs, time and efforts involved in surgery.
\end{abstract}

Keywords: Micro-TESE, TESE, Azoospermia, Sperm retrieval, ICSI

\section{Background}

Nonobstructive azoospermia (NOA) is a condition characterized by absence of sperm in the ejaculate due to impaired spermatogenesis. Nonobstructive azoospermia is reported in about $60 \%$ of azoospermic patients and $15 \%$ of all infertile men [1]. The histologic patterns associated with NOA include Sertoli cell-only syndrome (SCOS), maturation arrest (MA), hypospermatogenesis and sclera-hyalinosis. Nonobstructive azoospermic men could benefit from surgical sperm retrieval and assisted conception by intracytoplasmic

\footnotetext{
* Correspondence: costantino.leonardo@gmail.com

'Department Gynaecological-Obstetrical and Urological Sciences, Sapienza

University, via del Policlinico n 155 cap, 00161 Rome, Italy

Full list of author information is available at the end of the article
}

sperm injection (ICSI). The goal of surgical sperm recovery is to retrieve an adequate number of sperm for ICSI.

Different approaches were used with the intention of increasing the chances of finding viable sperm in NOA patients and, at the same time, optimizing organ preservation [2-4]. The recent updated European Association of Urology (EAU) guidelines (2014) recommend testicular biopsy as the best procedure to provide a histological diagnosis and to find sperm. However, the same EAU guidelines are unclear as to the type of sperm retrieval procedure considered best and recommended for patients with NOA.

In 1999, Schlegel [5] reported a novel microsurgical method for testicular sperm extraction (microdissection 
TESE, or micro-TESE). The author introduced this technique with the aim of improving sperm recovery and reducing invasivity of TESE in patients with NOA.

During micro-TESE, with the use of an operating microscope, it is possible to identify and selectively extract larger seminiferous tubules which have higher probability of harboring spermatozoa.

A number of studies suggest that micro-TESE should become the standard in the management of men with NOA [5-9]. Micro-TESE appears to improve the frequency of successful sperm retrieval in NOA patients, despite the removal of dramatically less testicular tissue. Relevantly, the extraction of seminiferous tubules during micro-TESE does not compromise the subtunical blood vessels, therefore the testicular damage is reduced as compared to a standard TESE [5, 9-13]. Schlegel [5] reported an improvement of sperm retrieval from a rate of $45 \%$ with conventional TESE to a rate of $63 \%$ with micro-TESE. However, the literature reports acceptable recovery rates with different techniques including single TESE biopsy: 41.6-49.5 \% [14, 15]; multiple conventional TESE: 52.5-56 \% [16-18]; microTESE: $35-77 \%$ [16, 19-24].

A conventional TESE biopsy with a single small incision is minimally invasive. A multiple conventional TESE with many superficial testicular incisions and tissue extractions is more invasive and can cause damage particularly to the testicular subtunical vessels. MicroTESE could also entail some damage to testis: it requires an extended equatorial incision to expose the parenchyma. Some concern has recently been raised on the risk of hormonal impairment after micro-TESE due to testicular damage $[12,25,26]$. In addition, micro-TESE requires the need of specific surgical equipment and skills with increased costs and operative time.

Following our preliminary experience [27], in the present study we performed testicular sperm retrieval in NOA patients using a novel surgical approach consisting in steps of increasing invasiveness: we refer to this procedure as to "stepwise micro-TESE". Our aim was to evaluate the performance of TESE versus micro-TESE by comparing the two methods on the same testicles in terms of sperm retrieval. We also provide the clinical outcome after ICSI.

\section{Methods}

\section{Study population}

In the present study, from March 2007 to April 2013, 343 NOA patients were referred for sperm retrieval at our fertility clinic. Azoospermia was diagnosed when the absence of sperm was observed in two semen samples after $600 \mathrm{~g}$ centrifugation and screening at 400x magnification using an inverted microscope, according to the
World Health Organization guidelines [28]. Surgical sperm retrieval was performed either by means of single or multiple conventional TESE or by micro-TESE. All the procedures were performed by a single expert surgeon (GF). In March 2007, it was decided that all future micro-TESE would have been performed with the new stepwise micro-TESE approach, consisting in a threesteps biopsy, as described later. Indications for stepwise micro-TESE were previous unsuccessful TESE/microTESE, unfavorable histology (complete SCOS or MA) and/or Klinefelter syndrome (KS).

Sixty-four patients received stepwise micro-TESE. Information collected included a clinical history and examination: patient's age was $35.4 \pm 5.07$; levels of serum FSH, luteinizing hormone (LH) and inhibin B were respectively $25.8 \pm 12.45,10.5 \pm 6.57$ and $19.7 \pm 17.67$. The etiology of azoospermia was defined for 38 patients whereas in the remaining 26 it was undefined: 25 were Klinefelter, 12 had a history of chriptorchidism and 1 with micro deletion of the AZFc. All patients were tested for Y-microdeletions. No patient with AZFa, AZFb, AZFab, AZFbc, AZFabc microdeletions were included in our stud group since it is our policy to discourage surgery (TESE or microTESE) in patients with these conditions due to the known no chance of success in sperm retrieval. Twenty-three out of the 64 patients with azoospermia had undergone previous unsuccessful sperm retrieval. Of the 64 patients, 2 (3.1 \%) had a testicular volume $>12 \mathrm{ml} ; 9(14.1 \%)$ had a volume between 6 and $12 \mathrm{ml}$, and $53(82.82 \%)$ had a severely reduced volume $(<6 \mathrm{ml})$. No pre-operative hormonal treatment was planned in any patients; in particular, none of the KS men received testosterone replacement prior to surgery.

\section{Surgical technique}

The surgical procedure was performed under general anesthesia. After scrotal disinfection with iodopovidone and clorexidine digluconate, the spermatic cord and the scrotal skin were infiltrated with $8 \mathrm{ml}$ of $7.5 \mathrm{mg} / \mathrm{ml}$ ropivacaine hydrochloride (Norepine, ASTA, Milan, Italy). The testicle on which the procedure was started was the one with larger volume or, in case no difference was evident between the two testicles, the procedure began on the right one, assuming that varicocele, if present, is on the left side. The scrotum was incised longitudinally for $2 \mathrm{~cm}$ on the median raphe and the testis was then delivered through the incision. The stepwise micro-TESE method consisted in three steps: for the initial TESE step, a small $(5 \mathrm{~mm})$ equatorial horizontal incision of the albuginea with extrusion of the testicular parenchima and scissors biopsy of approximately $5 \times 2 \times 3 \mathrm{~mm}$ (Fig. 1a). The second step consisted in a micro-TESE: under an operative microscope (10-24X magnification; 
Carl Zeiss, OPMI Surgical Microscope, Germany), an equatorial bilateral extension of the original incision was performed up to the hilum, with attention to preserving subtunical vessels. The testicle was then split open bluntly and tubules were retrieved with jewellers forceps from different sites of the two testicular sections (20 or more) aiming to locate and collect the larger ones with an increased chance of harboring spermatozoa (Fig. 1b); no attempt was made to retrieve tubules in the deep
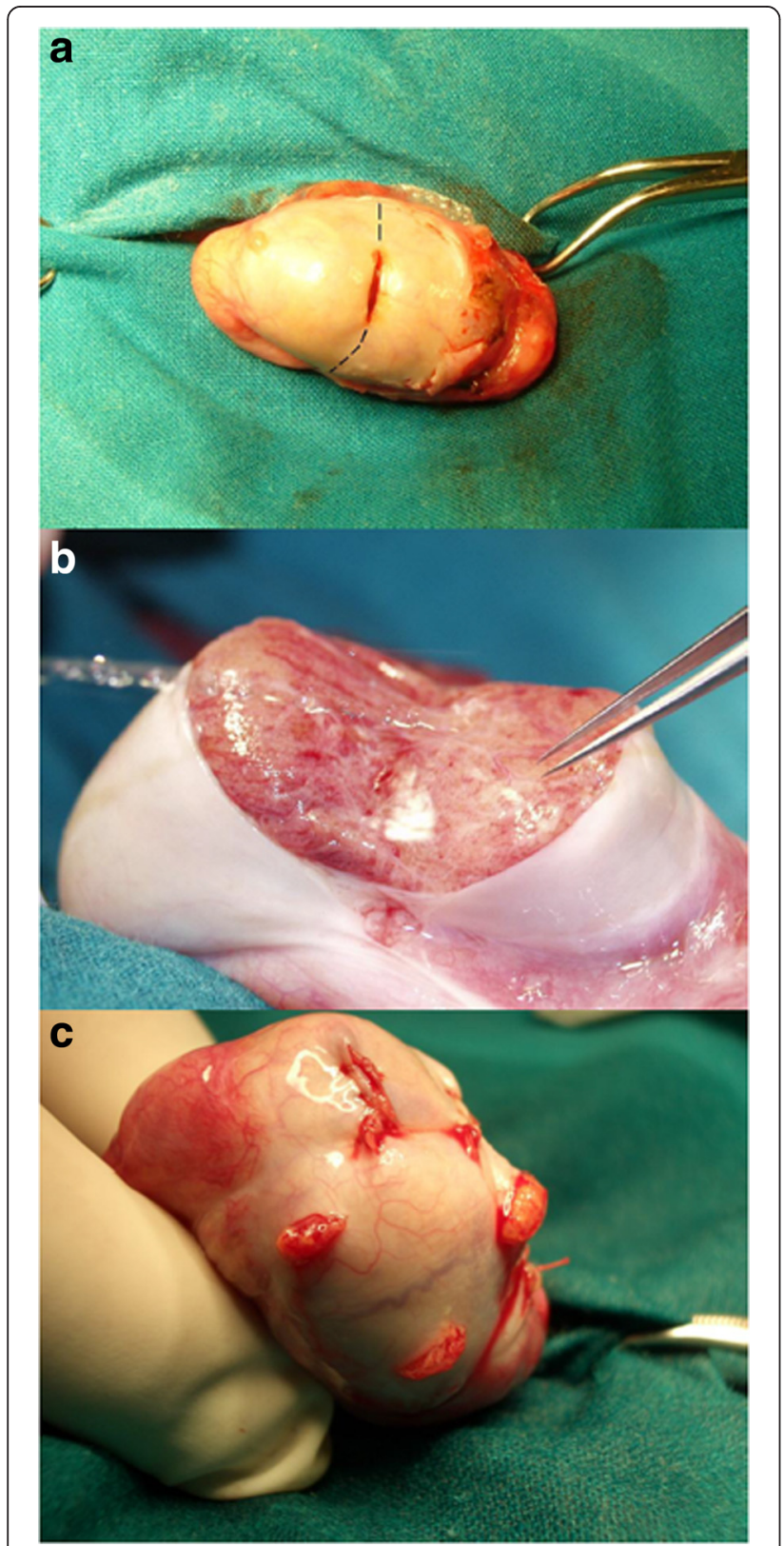

Fig. 1 Stepwise microTESE; a Conventional single TESE: the dotted line indicates the extension of the original incision in order to perform micro-TESE; $\mathbf{b}$ micro-TESE; c contralateral conventional multiple TESE testicular parenchima [29]. At the end of the procedure, the albuginea incision was closed with a VICRYL 5/0 running suture. If no sperm was found so far, we proceeded with the third step: multiple conventional superficial biopsies (4-8 sampling) on the contralateral testicle (Fig. 1c). Each one of the multiple biopsies of the third step had identical procedure and sample size as described for the initial single TESE step. The surgical procedure was always performed by the same surgeon (GF) and the specimen processed by the same biological team.

For hystological examinations, a fragment of testicular parenchyma removed in the first TESE step was washed in buffered medium (Quinn's Advantages Medium with HEPES, SAGE, Cooper Surgical, Pasadena, USA) with 2.5 \% Human Serum Albumin (HSA, Albutein, Alpha Therapeutic Milan, Italy), fixed in Bouin's solution $(1 \mathrm{ml})$ and sent to the pathology laboratory. All histological examinations were performed by the same pathologist. The overall mean operating time was $1 \mathrm{~h} 30^{\prime} \pm 30^{\prime}$. In our hands, mean operating time for micro-TESE is approximately $1 \mathrm{~h} \mathrm{30}$, while for single conventional TESE and for multiple TESE it is approximately $20^{\prime}$ and $45^{\prime}$ respectively.

The sequence of events during stepwise micro-TESE involves contemporarily the surgery room and the laboratory: starting from one testicle, the surgeon extracts the first tissue fragment (TESE) and places it in a Petri dish (containing 6-8 $\mathrm{ml}$ of buffered medium with HSA) which is immediately sent to the adjacent laboratory. Here, a biologist opens up the seminiferous tubules by mechanically dissecting the tissue with glass coverslides (wet preparation) as previously described [30]. The sperm search on the wet preparation is performed simultaneously by two biologists on two separate inverted microscopes (NIKON Eclipse S) at 400x magnification. After approximately 10-15 min, whether or not sperm are found in the wet preparation, the TESE sample is centrifuged (600G for $10 \mathrm{~min}$ ). Part of the cell suspension is smeared and covered with mineral oil so that sperm search can continue on a more concentrated sample by one or more biologists simultaneously (for more severe cases even three or four biologists). If sperm is present, evaluation (motility, morphology) and quantification are provided. In parallel, after the initial tissue biopsy, the surgeon proceeds with micro-TESE. The microtubules extracted with micro-TESE are collected in a Petri dish and roughly shredded with coverslides as done for the initial TESE sample. If sperm are found in the two initial steps the contralateral biopies are avoided. Sperm search in the smeared samples can last even for 4-6 h, long after the surgery procedure is concluded. If sperm is not found on the day of surgery, the search continues on the next days for $1-2 \mathrm{~h}$ on the remaining 
cell suspensions. When sperm is found, it is either used on fresh ICSI cycles or cryopreserved for future use [30]. The samples retrieved during the different steps of the procedure were always treated and frozen separately.

\section{Statistical analysis}

Statistical analysis was performed using the SPSS 12.0 software. Differences between groups of patients in medians for quantitative variables and differences in distributions for categorical variables were tested with the Kruskal-Wallis on way analysis of variance (ANOVA) and Chi-square test, respectively. Using multiple logistic regression with the enter method, variables evaluated in the univariate analysis were entered and investigated as predictors of sperm retrieval versus no sperm retrieval. The logistic regression analysis was carried out using data from patients for whom complete data were available. The variables considered for entry into the model included LH, FSH, Inhibin B, previous TESE (categorical variables). An alpha value of $5 \%$ was considered as threshold for significance. Data are presented as median (range), mean \pm standard deviation (SD). Odds ratios $(\mathrm{OR})$ and $95 \%$ confidence intervals $(\mathrm{CI})$ were calculated for the parameters in each group, no sperm retrieval as reference group.

\section{Results}

In 18 out of 64 patients we were able to retrieve testicular spermatozoa (sperm retrieval rate, SRR $=28.1 \%$ ). No significant differences in terms of sperm retrieval were observed between TESE and micro-TESE [18/64 patients for TESE versus 18/64 for micro-TESE (Chi square test, $p=1)]$. In all cases in which we retrieved sperm with micro-TESE we had previously retrieved sperm with the initial single TESE, either immediately in the wet preparation or with a longer search after centrifugation. On the other hand, when no sperm was found in the first procedure, none was found in the second micro-TESE nor in the contralateral testicle $(N=46 / 64)$.

Of the 18 patients with positive sperm retrieval, 11 received only the two initial steps on the first testicle sparing the contralateral biopsy due to the fact that sperm was immediately found in the first testis. In the other 7 patients, sperm was not immediately found in the first testis with the two initial procedures, therefore we proceeded anyway on the contralateral testicle: all of them eventually had positive sperm retrieval in both testis.

The hystological evaluation performed on the specimens obtained from our 64 patients was: 37/64 patients (57.8 \%) SCOS, 15/64 (23.4 \%) MA, and 12/64 (18.8\%) sclera-hyalinosis. The histological diagnosis of positive sperm retrieval patients was: $10 \mathrm{MA}, 1$ sclera-hyalinosis and 7 SCOS. The histological diagnosis of negative sperm retrieval patients was: $5 \mathrm{MA}, 11$ had sclerahyalinosis and $30 \mathrm{SCOS}$ (Table 4). A significant (Chi square test, $p=0.001$ ) higher sperm retrieval was obtained in patients with MA $(10 / 15=67 \%$ positive versus $5 / 15=33 \%$ negative) when compared to patients with $\operatorname{SCOS}(7 / 37=18.9 \%$ positive versus $30 / 37=81.1 \%$ negative) and sclera-hyalinosis $(1 / 12=0.8 \%$ positive versus $11 / 12=99.2 \%$ negative, Table 1 ). However, due to the small numbers of patients in each class, the statistical analysis has a reduced power.

The mean age of 18 men with positive sperm retrieval was 34.2 \pm 7.16 ; mean FSH level was $25.9 \pm 15.10$, mean LH level was $12.5 \pm 9.40$ and mean inhibin B level was $14.9 \pm 5.63$. On the other hand, in the 46 with negative sperm retrieval, mean age was $35.9 \pm 3.94$. Average levels of FSH, LH and inhibin B were $25.7 \pm 11.60,9.7 \pm 4.88$ and $20.0 \pm 18.86$, respectively. No significant differences for each of these variables were observed between patients with positive and negative sperm retrieval (Table 2). Twenty-three patients had previously undergone a TESE elsewhere, and for 6 of them stepwise micro-TESE resulted in a positive sperm retrieval (26\%). On the other hand, of the 41 patients who had not previously undergone a TESE elsewhere, 12 had positive sperm retrieval with step-wise micro-TESE (29\%; Chi square test, NS; Table 3). With a multiple logistic regression the predictive value of serological markers (FSH, LH and inhibin B) and of a previous unsuccessful TESE was investigated (Table 4). No significant pre-operative predictors of sperm retrieval, including previous TESE or serological markers, were observed at univariate or multivariate analysis.

Stepwise micro-TESE was always optimally tolerated by patients with minimal post-operative pain and no major complications. Fifteen out of the 18 patients with positive sperm retrieval had sperm cryopreservation whereas 3 of them underwent an ICSI cycle with fresh sperm. Our results in terms of fertilization and live birth rate are comparable with those in the literature. Overall, 2 patients (1 KS with AZFc deletion and 1 SCOS) had unsuccessful fertilization both with cryopreserved sperm. Four patients had embryo transfer with negative

Table 1 Histological diagnosis of patients with positive or negative sperm retrieval after stepwise micro-TESE. Significantly higher sperm retrieval was obtained in patients with MA when compared to patients with SCOS and sclera-hyalinosis

\begin{tabular}{llll}
\hline Histology & $\begin{array}{l}\text { Positive sperm } \\
\text { retrieval }\end{array}$ & $\begin{array}{l}\text { Negative sperm } \\
\text { retrieval }\end{array}$ & Chi square \\
\hline MA (15 cases) & $10 / 15(67 \%)$ & $5 / 15(33 \%)$ & $P=0.001$ \\
SCO (37 cases) & $7 / 37(18.9 \%)$ & $30 / 37(81.1 \%)$ & \\
Sclera-hyalinosis (12 cases) & $1 / 12(0.8 \%)$ & $11 / 12(99.2 \%)$ & \\
\hline
\end{tabular}


Table 2 Mean Age and preoperative variables (FSH, LH and inhibin B) in patients with positive or negative sperm retrieval after stepwise micro-TESE: none of these variables was predictive of sperm retrieval

\begin{tabular}{llll}
\hline & Positive & Negative & $p$ \\
\hline$N$ & 18 & 46 & \\
Mean age & $34.2 \pm 7.16$ & $35.9 \pm 3.94$ & 0.191 \\
FSH & $25.9 \pm 15.10$ & $25.7 \pm 11.60$ & 0.525 \\
LH & $12.5 \pm 9.40$ & $9.7 \pm 4.88$ & 0.586 \\
Inhibin B & $14.9 \pm 5.63$ & $20.0 \pm 18.86$ & 0.887 \\
\hline
\end{tabular}

beta-HCG (1 MA and 1 SCOS with cryopreserved sperm; 2 MA with fresh sperm). One SCOS patient had positive beta-HCG with cryopreserved sperm but no heartbeat was observed. One MA patient underwent an ICSI cycle with fresh sperm resulting in abortion at 8 weeks of gestation. Finally 3 ICSI cycles (all MA with cryopreserved sperm) ended with 4 babies delivered: 1 twin ( 1 female and 1 male) and 2 singleton ( 2 males) pregnancies (livebirth rate $27.2 \%=3 / 11$ ICSI cycles).

\section{Discussion}

In this study, a novel stepwise micro-TESE technique was performed on 64 patients with severe NOA undergoing testicular sperm extraction. First, a single TESE sample was taken from one testicle and, after this, a micro-TESE was performed extending the same testicular incision. The third step consisted in contralateral conventional multiple biopsies in case of negative sperm retrieval on the first testis. The rationale behind our study design was to explore the possibility of a gradual sperm retrieval approach aiming to minimize invasiveness, and to compare the efficiency of micro-TESE with conventional TESE. In our fertility center, surgery proceeds in parallel with sperm search in the laboratory and we are used to move to the contralateral testis in all cases of negative sperm retrieval, in order to maximize the chance of success. The contralateral multiple TESE served also as an additional control that micro-TESE was correctly performed in the first testis. Unexpectedly, no differences were seen in SRR among the initial TESE, the following micro-TESE and the final contralateral multiple TESE.

Conventional multiple TESE consists in random incisions which may result in atrophy and devascularization of the surrounding testicular tissue. This effect, together

Table 3 Sperm retrieval rate was similar in the groups of patients who underwent or not a previous unsuccesful TESE

\begin{tabular}{llll}
\hline & Previous TESE & No previous TESE & Chi square \\
\hline Positive sperm retrieval & $6 / 23(26 \%)$ & $12 / 41(29 \%)$ & $P=0.552$ \\
\hline
\end{tabular}

Table 4 Multiple logistic regression with the enter method. Variables evaluated in the univariate analyses were entered and investigated as predictors of sperm retrieval versus no sperm retrieval

\begin{tabular}{lllll}
\hline & OR & $95.0 \%$ C.I. & & $P$ \\
\hline FSH & 0.979 & 0.863 & 1.112 & 0.749 \\
LH & 1.035 & 0.829 & 1.293 & 0.760 \\
Inhibin B & 0.944 & 0.787 & 1.132 & 0.532 \\
Previous TESE & 0.257 & 0.014 & 4.584 & 0.356 \\
\hline
\end{tabular}

with the intratesticular bleeding and scar formation, can easily damage the spermatogenetic pathway and hormone production [8]. Micro-TESE with microtubules extraction from superficial sites of the section is less invasive than conventional multiple TESE [5]. This method, thanks to the optical magnification, allows a sparing of subtunical albuginea and intratesticular vessels with a minimal excision of testicular parenchyma. In fact, single seminiferous tubules can be collected without impairing the surrounding tissues. In the literature, there are many reports indicating micro-TESE as a more efficient method to retrieve spermatozoa with reasonable scarce invasiveness when compared to multiple biopsies. Tsujimura and collegues [13] reported a SRR of $45 \%$ obtained with salvage micro-TESE performed after previous failed conventional TESE. Overall, the reported rates of successful sperm retrieval with micro-TESE varies between 47 and $66 \%[7,8,12,31,32]$. However, in our view, it is reasonable to believe that many of these successful micro-TESE cases might have benefited from a less invasive approach of sperm retrieval. In these studies, micro-TESE was apparently offered to all NOA patients, even those with good prognosis. For instance, the operated population often included NOA patients with the histologic pattern of hypospermatogenesis, although it is well known that SRR in this situation is very high with any sperm retrieval technique [33].

We operate in a private fertility center where microTESE, due to its higher costs (approximately $50 \%$ higher than for conventional TESE) is offered only to NOA patients with severe clinical diagnosis and prognosis (complete SCOS or MA, KS, previous unsuccessful TESE). This might be an explanation why our overall SRR of $28.1 \%(18 / 64)$ is low when compared to those reported in the literature. However, it should be noticed that in other reports, when looking only to NOA subpopulations with severe prognosis, a similarly low SRR was reported, as described in a detailed review from Ghalayini et al. [24].

In contrast with what expected, our micro-dissection step did not improve the chance of finding sperm: the SRR obtained with micro-TESE or with the initial single 
conventional TESE coincided. Moreover, the multiple TESE performed on the contralateral testicle always confirmed the outcome obtained on the first testicle, either successful or not.

A possible reason for our micro-TESE not improving SRR could be that our tubule collection method during micro-TESE remained superficial. Ramasamy and colleagues [29] have recently reported their technique of micro-TESE starting with a superficial extraction of tubules followed by a deeper and more extensive search below the superficial section. This second step improved the SRR by $18.4 \%$ [29]. It is reasonable to assume that by extending our tubules collection to the deeper part of the testis, our SRR could be improved as well. However, one must take into account that an extensive procedure might entail additional damage and significantly prolong surgical time [34].

In another study from Marconi and colleagues [32], four surgical methods were compared, namely unifocal conventional TESE, unifocal micro-TESE, trifocal conventional TESE and trifocal conventional TESE plus micro-TESE. Consistently with our results, no difference was observed between micro-TESE and trifocal conventional TESE. Only the combination of trifocal conventional TESE plus unifocal micro-TESE significantly increased the SRR when compared to unifocal conventional TESE.

A surgical approach similar to ours has been applied by Turunc and colleagues [31]. The authors performed exclusively micro-TESE in a subgroup of severe NOA patients with testicular atrophy, obtaining a SRR of approximately $20 \%$. Another larger group of patients with a less severe prognosis, underwent firstly a trifocal conventional TESE. Only when no sperm was found, microTESE was performed by joining the three original incisions, therefore increasing the SRR from about $34 \%$ up to $51 \%$. Anyway, it has to be pointed out that the majority of successes were obtained with the initial conventional TESE. These data support our belief that microTESE should not be offered indiscriminately to all NOA patients but a gradual approach should be warranted.

A large proportion of our population was represented by men with KS $(25 / 64=39.1 \%)$. In our view this condition is one of the most severe forms of NOA [30]. Of these 25 patients, 6 had positive sperm retrieval (24\%). Theoretically, micro-TESE should be an ideal approach for men with KS who are characterized by small testes, extensive sclera-hyalinosis and scattered areas of remaining tubules. In this situation, the magnification system used during micro-TESE helps in identifying the tubules among the sclerotic tissue. The SRR of KS man in the present series resulted considerably low when compared to other published studies, including one from our group (ref. 30). A possible explanation is that, in the present study group, the mean age of KS patients ( $35.5 \pm 5.18)$ was substantially higher than that reported in other studies [30, 35, 36]. It is in fact well known how sclera-hyalinosis increases and spermatogenesis declines with age in KS patients.

The histology in our patient's population was represented by SCOS (57.8 \%), MA (23.4\%) and sclerahyalinosis $(18.8 \%)$. The condition of hypospermatogenis, a less severe form of NOA, was not represented among our 64 patients. Interestingly, we observed a significantly higher SRR in patients with MA (Table 1) when compared to the other histological diagnosis, although our sample size is limited to obtain definitive conclusions. On the contrary, sclera-hyalinosis appeared to be related to a scarce chance of retrieving sperm. The literature is still controversial in terms of the prognostic value of testicular histology. Some authors report a higher success rate of micro-TESE in case of SCOS [21, 29]. This could be reasonably due to an incomplete SCOS, a condition in which larger microtubules with spermatogenesis can be easily identified with the optical magnification among those with only Sertoli cells. The histological diagnosis of our patients population can explain our low SRR in the present series: most of our patients had and unfavorable histology of SCOS (57.8 \%) and sclera-hyalinosis $(18.8 \%)$; only a smaller proportion had MA (23.4 \%) and nearly all of them had positive SRR (10/15).

In our experience, no significant pre-operative predictors of sperm retrieval, including previous TESE or serological markers, were observed at univariate or multivariate analysis (Tables 2, 3 and 4). Particularly, in our study, levels of FSH, LH, inhibin B were not predictive of sperm retrieval, consistently to the results reported elsewhere [37, 38]. In contrast, other groups reported a positive predictive power of some preoperative variables, such as FSH $(39 ; 17)$ and inhibin B $[39,40]$. Furthermore, in the subgroup of patients who had undergone a previous negative TESE elsewhere $(N=23, \mathrm{SRR}=26 \%)$, the SRR did not differ from the subgroup of patients who had not received a previous negative TESE $(N=41, \mathrm{SRR}=29 \%$, Table 3$)$.

\section{Conclusions}

Our study indicate that 1) in patients with poor prognosis NOA even micro-TESE did not improve the chance of retrieving sperm; 2) in all patients with successful sperm retrieval, the initial, less invasive single conventional biopsy would have been enough to obtain sperm; 3) micro-TESE was always optimally tolerated by patients and left minimal if no scars; 4) due to the priority that should be always given to organ preservation, we believe that the gradual approach of stepwise microTESE could be ideal for testicular sperm retrieval in all NOA men. In this way costs, time and efforts involved in the surgery procedure would be drastically reduced. 


\section{Ethics approval and consent to participate} The study was approved by the Institutional Ethics Committee of European Hospital. All patients were informed on the aspects of the surgical procedure and signed a written informed consent.

\section{Consent for publication \\ Not applicable.}

\section{Availability of data and materials}

All the data supporting our findings is contained within the manuscript.

\section{Abbreviations}

AZF: azoospermia factor; beta-HCG: beta human chorionic gonadotropin; EAU: European Association of Urology; FSH: follicle stimulation hormone; HSA: Human Serum Albumin; ICSI: intracytoplasmic sperm injection; KS: Klinefelter syndrome; LH: luteinizing hormone; MA: maturation arrest; micro-TESE: microdissection testicular sperm extraction; NOA: nonobstructive azoospermia; SCOS: Sertoli cell-only syndrome; SRR: sperm retrieval rate; TESE: testicular sperm extraction.

\section{Competing interest}

The authors declare that they have no competing interests.

\section{Authors' contributions}

GF and EG conceived the study concept and design. FS and VC carried out acquisition of data and drafting the manuscript. CDD and CL carried out analysis and interpretation of the data. DD and MGM carried out drafting of the manuscript. PFG and AG carried out Critical revision of the manuscript. $\mathrm{CDD}$ and $\mathrm{CL}$ carried out statistical analysis. All Authors read and approve the final version of the manuscript.

\section{Acknowledgments}

The authors would like to thank to Dr Mario Terribile, PhD, for his precious help during data acquisition evaluation and discussion.

\section{Funding}

Not applicable.

\section{Author details}

'Department Gynaecological-Obstetrical and Urological Sciences, Sapienza University, via del Policlinico n 155 cap, 00161 Rome, Italy. ${ }^{2}$ Centre for Reproductive Medicine, European Hospital, Rome, Italy. ${ }^{3}$ Department Urology, Sant' Andrea Hospital, Sapienza University, Rome, Italy. ${ }^{4}$ Robotic Urology Department, Policlinico Abano Terme, Padova, Italy.

\section{Received: 28 September 2015 Accepted: 1 May 2016}

Published online: 12 May 2016

\section{References}

1. Dabaja AA, Schlegel PN. Microdissection testicular sperm extraction: an update. Asian J Androl. 2013;15:35-9.

2. Oliveira Filho AB, Souza RS, Azeredo-Oliveira MT, Peruquetti RL, Cedenho AP. Microdissection testicular sperm extraction causes spermatogenic alterations in the contralateral testis. Genet Mol Res. 2010;9:1405-13.

3. Glina S, Soares JB, Antunes Jr N, Galuppo AG, Paz LB, Wonchockier R. Testicular histopathological diagnosis as a predictive factor for retrieving spermatozoa for ICSI in non-obstructive azoospermic patients. Int Braz J Urol. 2005:31:338-41.

4. Van Peperstraten A, Proctor ML, Johnson NP, Philipson G. Techniques for surgical retrieval of sperm prior to intracytoplsmic sperm injecyion (ICSI) for azoospermia. Cochrane Database Syst Rev. 2008;(2):CD002807.

5. Schlegel PN. Testicular sperm extraction: microdissection improves sperm yield with minimal tissue excision. Hum Reprod. 1999;14:131-5.

6. Silber SJ. Microsurgical TESE and the distribution of spermatogenesis in non-obstructive azoospermia. Hum Reprod. 2000;15:2278-84.
7. Amer M, Ateyah A, Hany R, Zohdy W. Prospective comparative study between microsurgical and conventional testicular sperm extraction in non-obstructive azoospermia: follow-up by serial ultrasound examinations. Hum Reprod. 2000;15:653-6.

8. Ramasamy R, Yagan N, Schlegel PN. Structural and functional changes to the testis after conventional versus microdissection testicular sperm extraction. Urology. 2005;65:1190-4.

9. Ramasamy R, Ricci JA, Palermo GD, Gosden LV, Rosenwaks Z, Schlegel PN. Successful fertility treatment for Klinefelter's syndrome. J Urol. 2009;182:1108-13.

10. Okada H, Dobashi M, Yamazaki T, Hara I, Fujisawa M, Arakawa S, et al. Conventional versus microdissection testicular sperm extraction for nonobstructive azoospermia. J Urol. 2002;168:1063-7.

11. Okubo K, Ogura K, Ichioka K, Terada N, Matsuta Y, Yoshimura K, et al. Testicular sperm extraction for non-obstructive azoospermia: results with conventional and microsurgical techniques. Hinyokika Kiyo. 2002;48:275-80.

12. Tsujimura A, Matsumiya K, Miyagawa Y, Tohda A, Miura H, Nishimura K, et al. Conventional multiple or microdissection testicular sperm extraction: a comparative study. Hum Reprod. 2002;17:2924-9.

13. Tsujimura A, Miyagawa Y, Takao T, Takada S, Koga M, Takeyama M, et al. Salvage microdissection testicular sperm extraction after failed conventional testicular sperm extraction in patients with nonobstructive azoospermia. J Urol. 2006;175:1446-9.

14. Vernaeve V, Verheyen G, Goossens A, Van Steirteghem A, Devroey P, Tournaye $\mathrm{H}$. How successful is repeat testicular sperm extraction in patients with azoospermia? Hum Reprod. 2006;21:1551-4.

15. Donoso $P$, Tournaye $H$, Devroey $P$. Which is the best sperm retrieval technique for non-obstructive azoospermia? A systematic review. Hum Reprod Update. 2007:13:539-49.

16. Colpi GM, Piediferro G, Nerva F, Giacchetta D, Colpi EM, Piatti E. Sperm retrieval for intra-cytoplasmic sperm injection in non-obstructive azoospermia. Minerva Urol Nefrol. 2005;57:99-107.

17. Colpi GM, Colpi EM, Piediferro G, Giacchetta D, Gazzano G, Castiglioni FM, et al. Microsurgical TESE versus conventional TESE for ICSI in non-obstructive azoospermia: a randomized controlled study. Reprod Biomed Online. 2009;18:315-9.

18. Bromage SJ, Falconer DA, Lieberman BA, Sangar V, Payne SR. Sperm retrieval rates in subgroups of primary azoospermic males. Eur Urol. 2007;51:534-9.

19. El-Haggar S, Mostafa T, Abdel Nasser T, Hany R, Abdel HA. Fine needle aspiration vs. mTESE in non-obstructive azoospermia. Int J Androl. 2008;31:595-601.

20. Talas H, Yaman O, Aydos K. Outcome of repeated micro-surgical testicular sperm extraction in patients with non-obstructive azoospermia. Asian J Androl. 2007;9:668-73.

21. Esteves SC, Verza S, Prudencio C, Seol B. Sperm retrieval rate (SRR) in nonobstructive azoospermia (NOA) are related to testicular histopathology results but not to the etiology of azoospermia. Fertil Steril. 2010;94:S132

22. Esteves SC, Miyaoka R, Agarwal A. Surgical treatment of male infertility in the era of intracytoplasmic sperm injection - new insights. Clinics. 2011;66:1463-77.

23. Ravizzini P, Carizza C, Abdelmassih V, Abdelmassih S, Azevedo M, Abdelmassih R. Microdissection testicular sperm extraction and IVF-ICSI outcome in nonobstructive azoospermia. Andrologia. 2008;40:219-26.

24. Ghalayini IF, Al-Ghazo MA, Hani OB, Al-Azab R, Bani-Hani I, Zayed F, et al. Clinical comparison of conventional testicular sperm extraction and microdissection techniques for non-obstructive azoospermia. J Clin Med Res. 2011;19:124-31.

25. Takada S, Tsujimura A, Ueda T, Matsuoka Y, Takao T, Miyagawa Y, et al Androgen decline in patients with nonobstructive azoospemia after microdissection testicular sperm extraction. Urology. 2008;72:114-8.

26. Tsujimura A. Microdissection testicular sperm extraction: prediction, outcome, and complications. Int J Urol. 2007;14:883-9.

27. Franco G. Sperm retrieval in the azoospermic patient. In: Greco E, editor. Male infertility and ART. Firenze: Pacini Editore Press; 2008. p. 50-6.

28. World Health Organization. WHO Laboratory manual for the examination and processing of human semen and Sperm-Cervical Mucus and interaction. Cambridge: WHO; 2010.

29. Ramasamy R, Reifsnyder JE, Husseini J, Eid PA, Bryson C, Schlegel PN. Localization of sperm during microdissection testicular sperm extraction in men with nonobstructive azoospermia. J Urol. 2013;189:643-6. 
30. Greco E, Scarselli F, Minasi MG, Casciani V, Zavaglia D, Dente D, et al. Birth of 16 healthy children after ICSI in cases of nonmosaic Klinefelter syndrome. Hum Reprod. 2013;28:1155-60.

31. Turunc T, Gul U, Haydardedeoglu B, Bal N, Kuzgunbay B, Peskircioglu L, et al. Conventional testicular sperm extraction combined with the microdissection technique in nonobstructive azoospermic patients: a prospective comparative study. Fertil Steril. 2010;94:2157-60.

32. Marconi M, Keudel A, Diemer T, Bergmann M, Steger K, Schuppe HC, et al. Combined trifocal and microsurgical testicular sperm extraction is the best technique for testicular sperm retrieval in "low-chance" nonobstructive azoospermia. Eur Urol. 2012;62:713-9.

33. Tournaye H, Liu J, Nagy PZ, Camus M, Goossens A, Silber S, et al. Correlation between testicular histology and outcome after intracytoplasmic sperm injection using testicular spermatozoa. Hum Reprod. 1996;11:127-32.

34. Ramasamy R, Fisher ES, Ricci JA, Leung RA, Schlegel PN. Duration of microdissection testicular sperm extraction procedures: relationship to sperm retrieval success. J Urol. 2011;185:1394-7.

35. Plotton I, Giscard d'Estaing S, Cuzin B, Brosse A, Benchaib M, Lornage J, et al. Preliminary results of a prospective study of testicular sperm extraction in young versus adult patients with nonmosaic 47, XXY Klinefelter syndrome. J Clin Endocrinol Metab. 2015;100(3):961-7.

36. Sabbaghian M, Modarresi T, Hosseinifar H, Hosseini J, Farrahi F, Dadkhah F, et al. Comparison of sperm retrieval and intracytoplasmic sperm injection outcome in patients with and without Klinefelter syndrome. Urology. 2014;83(1):107-10

37. Raheem A, Garaffa G, Rushwan N, De Luca F, Zacharakis E, Abdel Raheem T, et al. Testicular histopathology as a predictor of a positive sperm retrieval in men with non-obstructive azoospermia. BJU Int. 2013;111:492-9.

38. Gul U, Turunc T, Haydardedeoglu B, Yaycioglu O, Kuzgunbay B, Ozkardes H. Sperm retrieval and live birth rates in presumed Sertoli-cell-only syndrome in testis biopsy: a single centre experience. Andrology. 2013;1:47-51.

39. Bohring C, Schroeder-Printzen I, Weidner W, Krause W. Serum levels of inhibin B and follicle-stimulating hormone may predict successful sperm retrieval in men with azoospermia who are undergoing testicular sperm extraction. Fertil Steril. 2002;78:1195-8.

40. Brugo-Olmedo S, De Vincentiis S, Calamera JC, Urrutia F, Nodar F, Acosta AA. Serum inhibin $B$ may be a reliable marker of the presence of testicular spermatozoa in patients with nonobstructive azoospermia. Fertil Steril. 2001:76:1124-9.

\section{Submit your next manuscript to BioMed Central and we will help you at every step:}

- We accept pre-submission inquiries

- Our selector tool helps you to find the most relevant journal

- We provide round the clock customer support

- Convenient online submission

- Thorough peer review

- Inclusion in PubMed and all major indexing services

- Maximum visibility for your research

Submit your manuscript at www biomedcentral.com/submit

Biomed Central 